\title{
Effect of harmonic relatedness on the detection of temporal asynchronies
}

\author{
BARBARA TILLMANN and JAMSHED J. BHARUCHA \\ Dartmouth College, Hanover, New Hampshire
}

\begin{abstract}
Speeded intonation judgments of a target chord are facilitated when the chord is preceded by a harmonically related prime chord. The present study extends harmonic priming to temporal asynchrony judgments. In both tasks, the normative target chords (consonant, synchronous) are processed more quickly and accurately after a harmonically related prime than after a harmonically unrelated prime. However, the influence of harmonic context on sensitivity $\left(d^{\prime}\right)$ differs between the two tasks: $d^{\prime}$ was higher in the related context for intonation judgments but was higher in the unrelated context for asynchrony judgments. A neural net model of tonal knowledge activation provides an explanatory framework for both the facilitation in the related contexts and the sensitivity differences between the tasks.
\end{abstract}

A context influences the processing of an upcoming event. The processing of a target word is facilitated if the word is preceded by a semantically related word (Meyer $\&$ Schvaneveldt, 1971) or if it forms a semantically and syntactically congruent ending to a sentence (Stanovich $\&$ West, 1979). In music, the processing of a target chord is facilitated if the chord is preceded by a harmonically related context that can be short (one chord) or long (chord sequences). Harmonic priming has been studied by using judgments of the sensory dissonance or intonation of the target chord. In the present study, harmonic priming is investigated by using temporal asynchrony judgments to examine the generality of the priming phenomena across tasks.

The relationships between prime and target that have been manipulated in harmonic-priming studies are based on the regularities inherent in the Western tonal system. A brief review of the major regularities will allow us to present the underlying rationale of harmonic-priming research. In Western tonal music, a set of 12 chromatic tones is combined in a highly constrained way, yielding a system of relationships. The 12 tones are organized in subsets of 7 tones, called diatonic scales. On each degree of a scale, chords (combinations of 3 tones) can be constructed. The chords built on the first, fifth, and fourth scale degrees (referred to as the tonic, dominant, and subdominant chords, respectively) are more frequent in tonal musical pieces and have more central functions than do chords built on other degrees (Francès, 1958;

This research has been supported in part by a grant to B.T. from the Deutsche Akademische Austauschdienst DAAD and by grants to J.J.B. from the National Science Foundation (SBR-9601287) and NIH (2P50 NS17778-18). Correspondence concerning this article should be addressed to B. Tillmann, Université Claude Bernard Lyon I, Laboratoire Sciences \& Systèmes Sensoriels, Equipe Audition-CNRS-UMR 5020, 50 Avenue Tony Garnier, F-69366 Lyon cedex 07, France (e-mail: barbara.tillmann@olfac.univ-lyon1.fr).
Krumhansl, 1990). Identical chords may occur in a variety of different keys. Chords sharing a parent key are said to be harmonically related (such as the chords C-major and $\mathrm{B}$-major, which both belong to the key of F-major). Harmonically related chords are those that are more frequently associated in tonal musical pieces than are other chords. Through mere exposure, listeners of Western music have acquired implicit knowledge of the specific constraints and conventional relationships among tones, chords, and keys (Tillmann, Bharucha, \& Bigand, 2000). A musical context activates listeners' tonal knowledge so that they expect harmonically related events more than they expect harmonically unrelated events. These expectations then influence the processing of further events, as is shown by harmonic-priming studies with short and long contexts.

In harmonic-priming studies with short contexts (Bharucha \& Stoeckig, 1986, 1987; Tekman \& Bharucha, 1992, 1998), a single chord defined the prime and was followed by the target chord. The two chords were either related (shared a common key) or unrelated (did not share a common key) harmonically. For example, when the prime was a $\mathrm{C}$-major chord, a Bb-major chord was a related target, and a F\#-major chord was an unrelated target. On half the trials, the target chord was slightly mistuned. Participants were asked to make a speeded intonation judgment - that is, to decide as quickly and accurately as possible whether the target chord was in tune. The priming effect was shown by (1) a bias to judge target chords to be in tune when they were related to the prime and (2) shorter response times for in-tune targets when they were related to the prime and for out-of-tune targets when they were unrelated to it. Thus, a single chord can generate expectancies for related chords to follow, resulting in greater perceived consonance and faster processing for expected chords. Harmonic-priming effects have been extended to longer prime contexts (Bigand, Madurell, Tillmann, \& Pineau, 1999; Bigand \& Pineau, 1997; Till- 
mann, Bigand, \& Pineau, 1998). Bigand and Pineau manipulated the global context of eight-chord sequences in order to change the expectations for the last chord. The penultimate chord (the local context) was held constant in the context sequences to control for local psychoacoustic influences. Participants were faster and more accurate in their intonation judgment of the last chord when it was strongly related (the tonic chord of the context key) than when it was less related (the subdominant chord of the context key). These results suggest that harmonic priming involves higher level harmonic structures and does not occur only from chord to chord.

Several control conditions suggest that harmonic context effects are unlikely to be caused by sensory priming. In short contexts, harmonic priming occurs even if the target chord does not share tones (or even harmonics) with the prime or is preceded by white noise (Bharucha \& Stoeckig, 1987; Tekman \& Bharucha, 1992). In long contexts, harmonic priming persists even if the local context (up to six chords; Bigand et al., 1999, Experiment 3) is held constant or if the target chord itself never occurs in the preceding context (Bigand, Poulin, Tillmann, D'Adamo, \& Madurell, 2001). Harmonic priming thus occurs at a cognitive level, on the basis of the activation of listeners' implicit knowledge of the Western tonal system, with its underlying regularities and the hierarchies of importance of tonal events.

The observed harmonic-priming effects and their interpretation in terms of knowledge activation have always involved a judgment of the sensory dissonance of the target chord. For half of the trials, target chords were rendered either out of tune (Bharucha \& Stoeckig, 1986, 1987; Tekman \& Bharucha, 1992, 1998) or dissonant by adding a nondiatonic tone (Bigand et al., 1999; Bigand \& Pineau, 1997; Tillmann et al., 1998). Participants had to decide if the target chord was in tune (consonant) or out of tune (dissonant). It might be argued that the contextual dissonance created by the harmonically unrelated context creates a confound with the mistuning or dissonance of the target chord itself. In other words, the tendency to judge the target to be consonant and to respond more quickly in a harmonically related context may simply be a confounding of these two forms of dissonance, rather than being indicative of an underlying priming process in terms of knowledge activation (see Terhardt, 1984, for two kinds of dissonance). The goal of our study was to explore the generality of harmonic priming by extending it to a task based on a different perceptual judgment of the target chord: the detection of temporal asynchrony.

Studies of auditory perception have provided evidence for listeners' sensitivity to temporal asynchrony in simultaneously occurring events. The asynchrony in simultaneously occurring events is used for grouping or segregation of streams in order to determine sound sources in auditory scene analysis (Bregman, 1990). For example, two pure tones of different frequencies segregate in streams if their onsets are played relatively asynchronously but fuse into one percept for synchronous onsets. Zera and Green $(1993,1995)$ studied the perception of temporal asynchrony of a single harmonic at both the onset and the offset of a complex sound. Listeners' discrimination of an asynchronous complex from a synchronous one was superior when the asynchronous feature was situated at the onset of the complex sound. In musical performance, onset asynchronies and, in particular, early onsets in the melodic line (melody leads) provide important perceptual cues aiding listeners in identifying the melody in multivoiced pieces (e.g., Palmer, 1996).

For sequentially occurring events, the detection of deviations from temporal regularity has been investigated in sequences with regular interonset intervals made of clicks (Halpern \& Darwin, 1982) or tones (Drake, 1993; Drake \& Botte, 1983; Hirsh, Monahan, Grant, \& Singh, 1990) or in musical excerpts (Repp, 1992, 1998, 1999). The performance is influenced not only by the interonset interval size and the positions in the sequence (initial vs. final), but also by the sequence's structure in the vicinity of the to-be-detected event. The musical structure of the sequence interacts with the detection of temporally deviant events in isochronously played musical sequences. Across all positions in the musical sequence, the detection accuracy decreases in places where lengthening would typically occur in an expressive musical interpretation (e.g., at the end of a section). The musical structure of the previous context allows the development of expectations on timing structures for upcoming events that influence the detection accuracy of temporal deviations.

In our study, the influence of harmonic expectations on the detection of a temporal onset asynchrony in simultaneously occurring tones was investigated. As has been shown in previous priming studies, a harmonic context activates listeners' tonal knowledge and allows the development of expectations for subsequent chords, with harmonically related events being more strongly expected. In the present study, we were interested in the influence of harmonic relatedness of the context on the judgment of a temporal feature in a chord. The temporal asynchrony was related to the onset of one tone in a chord of four tones. In half of the trials, one tone of the target was played with a short temporal delay, and the participants were required to indicate whether the chord contained a temporal asynchrony.

\section{EXPERIMENT 1}

\section{Method}

Participants. Fifteen introductory psychology students participated in this experiment. Number of years of musical training, as measured by years of instrumental instruction, ranged from 0 to 13 , with a mean of $4(S D=4.24)$ and a median of 3 .

Material. Prime chords and target chords were major chords consisting of four component tones (i.e., C2-E3-G3-C4). Each of the 12 major chords occurred twice as target, preceded by either a 
related or an unrelated prime. The related prime chord was built on the degree of the chromatic scale that is seven semitones (a perfect fifth) above or five semitones (a perfect fourth) below (i.e., a frequency factor of $2^{7 / 12}$ above or $2^{5 / 12}$ below) the one for the target. The related pair formed an authentic cadence, a frequently occurring chord succession in Western tonal music that creates a strong impression of ending (e.g., G-major followed by C-major). The unrelated prime chord was built on a degree of the chromatic scale that is six semitones (i.e., a tritone, a frequency factor of $2^{7 / 12}$ ) above or below the one for the target, creating a pair with harmonically distant chords (e.g., $\mathrm{F} \#$-major followed by $\mathrm{C}$-major).

All the stimuli were played with sampled piano sounds produced by a KORG New SG-1D. The sound stimuli were captured by SoundEditPro software (MacroMedia), and the experiment was run on PsyScope software (Cohen, MacWhinney, Flatt, \& Provost, 1993). The prime chord sounded for $666 \mathrm{msec}$, the target chord sounded for $2 \mathrm{sec}$, and the interstimulus interval was set to 0 .

For prime chords and synchronous targets, the four tones of the chord were played at the same time. For the targets containing a temporal asynchrony (asynchronous targets), the tonic tone in the soprano voice (i.e., the highest tone in the chord) was played with a delay of $50 \mathrm{msec}$, as compared with the three other tones. This tone did not occur in either the related or the unrelated prime chord. Velocity, a parameter related to the sound level, was constant for all pitches (set to 64 in Performer 5.3 software, Marc of the Unicorn), except for the delayed tone, for which the velocity was adjusted for comparable salience (with a mean velocity of 62.3, ranging from 55 to 68). The calibration of the velocities was done on the basis of the agreement of two listeners.

Procedure. After a training phase on the experimental task with isolated chords, the 48 chord pairs were presented in random order. The participants had to make a normal versus one note late judgment on the second chord as quickly and accurately as possible by pressing one of two keys on the computer keyboard. The next trial began when the participant pressed a third key on the keyboard. In order to motivate the participants to respond as quickly and accurately as possible, the second chord stopped sounding immediately after a correct response (allowing the participants to continue with the next trial), but not after an incorrect response, which in addition, was followed by an alerting feedback signal.

\section{Results}

Percentages of errors ${ }^{1}$ (Figure 1, left panel) and response times for correct responses (Figure 1, right panel) were analyzed by two $2 \times 2$ analyses of variance (ANOVAs), with harmonic context (related/unrelated) and target type (synchronous/asynchronous) as within-subjects factors.

For the synchronous targets, error rates were lower and response times were faster for the related context than for the unrelated context. For asynchronous targets, error rates were higher and response times tended to be slower for the related context than for the unrelated context. This interaction between harmonic context and target type was significant for percentages of errors and for response times $\left[F(1,14)=7.58, M S_{\mathrm{e}}=220.4, p<.05\right.$, and $F(1,14)=9.64, M S_{\mathrm{e}}=1,812.47, p<.01$, respectively]. In addition, the effect of harmonic context was significant for percentages of errors $[F(1,14)=7.49$, $\left.M S_{\mathrm{e}}=50.10, p<.05\right]$. For response times, the size of the priming effect for synchronous targets (i.e., the difference in response times between related and unrelated contexts) was not correlated with years of musical training $[r(13)=.05]$. Overall, response times were faster for synchronous targets than for asynchronous targets $\left[F(1,14)=17.5, M S_{\mathrm{e}}=2,455.34, p<.01\right]$. It might be suggested that the presence of the critical task feature (the asynchronous tone) in the asynchronous targets allowed faster responding than for the synchronous targets; in other words, for synchronous targets, the participants may have waited somewhat longer before deciding that no late tone was forthcoming.
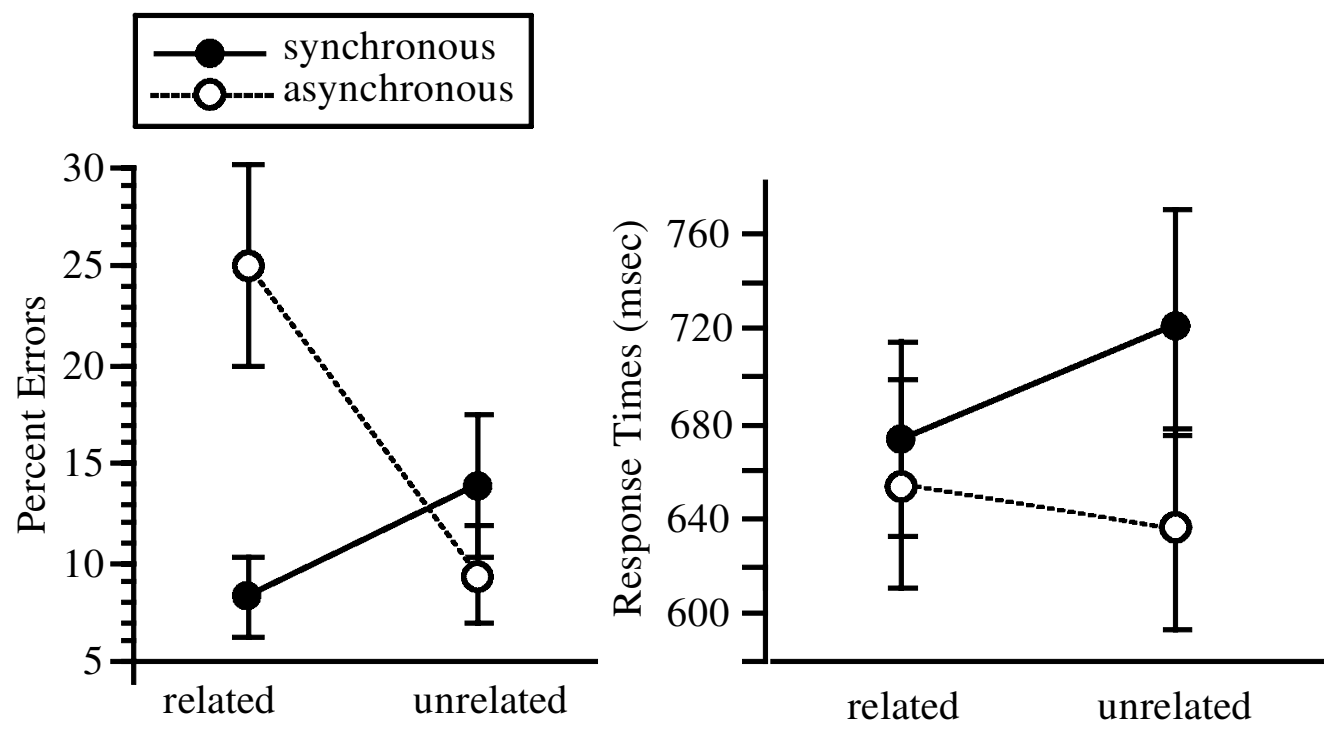

Figure 1. Percentages of errors (left panel) and correct response times (right panel) averaged across all chord pairs as a function of harmonic context (related/unrelated) and target type (synchronous/asynchronous) in Experiment 1. Error bars indicate between-subjects standard errors. 
Table 1

Mean Sensitivity $\left(d^{\prime}\right)$ and Mean Response Criterion $(c)$ for Experiment 1 (With Between-Subjects Standard Errors)

\begin{tabular}{|c|c|c|c|c|}
\hline \multirow[b]{3}{*}{ Parameter } & \multicolumn{4}{|c|}{ Harmonic Context } \\
\hline & \multicolumn{2}{|c|}{ Related } & \multicolumn{2}{|c|}{ Unrelated } \\
\hline & $M$ & $S E$ & $M$ & $S E$ \\
\hline$d^{\prime}$ & 2.16 & .19 & 2.50 & .17 \\
\hline$c$ & .30 & .10 & -.08 & .09 \\
\hline
\end{tabular}

Note-Positive values of $c$ stand for a tendency to respond normal; negative values stand for a tendency to respond one note late.

Two further analyses were performed in terms of signal detection theory, with sensitivity $\left(d^{\prime}\right)$ and response criterion $(c)$ as dependent variables and harmonic context as a within-subjects factor (Table 1). False alarms were defined as errors for synchronous targets, hits as correct responses for asynchronous targets. The parameters $d^{\prime}$ and $c$ were calculated for each participant separately. In cases without false alarms or with the maximum number of hits, frequencies of 0 and $N$ were converted into $1 / 2$ and $N-1 / 2$ (Kadlec, 1999). The possible range of $d^{\prime}$ scores was from 0 to 3.5. The response criterion $c$ was chosen because this bias parameter is independent of $d^{\prime}$ (Macmillan \& Creelman, 1991). The sensitivity $\left(d^{\prime}\right)$ was significantly higher for the unrelated context than for the related context $[F(1,14)=5.63, p<.05]$. The participants showed a small bias to answer one note late when the target was unrelated to the prime and to answer normal when it was related $[F(1,14)=7.47, p<.05]$.

\section{Discussion}

In Experiment 1, the harmonic relatedness of the context influenced the processing of a chord when a temporal synchrony judgment was required. A synchronous target chord (say, C-major) was faster and more accurately processed when it followed a chord belonging to the same musical key (G-major) rather than to another key (F\#-major). Chords with a temporal asynchrony showed the reverse pattern, with mainly the accuracy data being influenced by the harmonic context. Thus, the harmonic expectations that developed after the presentation of the prime chord influenced the processing of a temporal feature of the target chord. The results suggest that harmonic priming in short musical contexts generalizes beyond intonation judgments to temporal synchrony judgments. Previously reported facilitation effects in related contexts seem not to be based on a potential confound between contextual dissonance and dissonance of the target itself.

Analogous to the intonation task (Bharucha \& Stoeckig, 1986), a response bias was observed with temporal asynchrony detection in Experiment 1. The participants tended to respond synchronous for related chords and asynchronous for unrelated chords. A surprising result was the higher sensitivity (as measured by $d^{\prime}$ ) for unrelated than for related chords. In the intonation task, the reverse pattern has been reported for long prime contexts, with a sen- sitivity being higher for related than for unrelated chords (Tillmann \& Bigand, 2001; Tillmann et al., 1998).

In Experiment 2, this difference with single-chord prime contexts was further investigated by directly comparing the two tasks in a within-subjects design. The participants were required to judge the target chord for temporal asynchrony (normallone note late) in one phase of the experiment and for intonation (consonant/dissonant) in the other phase.

\section{EXPERIMENT 2}

\section{Method}

Participants. Twenty-eight introductory psychology students participated in this experiment; none had participated in Experiment 1 . Number of years of musical training, as measured by years of instrumental instruction, ranged from 0 to 16 , with a mean of $5.75(S D=3.52)$ and a median of 5 .

Material. The definition of related and unrelated chord pairs was identical to that in Experiment 1. A further methodological control was added in order to avoid possible temporal inaccuracies owing to the MIDI system: The same recordings of target chords were used in both harmonic contexts (following either a related or an unrelated prime). For the asynchrony task, the definitions of the synchronous and asynchronous targets were as described for Experiment 1 , with a mean velocity of the delayed tone of 63.8 (ranging from 58 to 68). For the intonation task, the consonant targets were exactly the same as the synchronous targets. For the dissonant targets, the sensory consonance was altered by adding an augmented fifth, played at reduced velocity in order to make the dissonance only moderately salient (i.e., C2-E3-G3-G\#3-C4). The velocity for the added tone was calibrated to produce comparable salience across chords (with a mean velocity of 43.2, ranging from 40 to 49 ).

Procedure. The experimental procedure was split into two phases. Each phase had the same structure as in Experiment 1. In one phase, the task was a temporal asynchrony judgment (nor$\mathrm{mal} /$ one note late); in the other phase, it was an intonation judgment (consonant/dissonant). The order of the two phases was counterbalanced over participants. As in Experiment 1, the next trial began when the participant pressed a third key on the keyboard, and the participants were alerted by a feedback signal if they gave an incorrect response.

\section{Results}

Accuracy. For each task (asynchrony/intonation), percentages of errors (Figure 2, left panels) were analyzed by a $2 \times 2$ ANOVA, with harmonic context and target type as within-subjects factors. For synchronous targets and for consonant targets, more errors were observed in the unrelated context than in the related context. For asynchronous targets and for dissonant targets, more errors were observed in the related context than in the unrelated context. This interaction between harmonic context and target type was significant for both tasks $[F(1,27)=$ $21.06, M S_{\mathrm{e}}=49.49, p<.001$, for the asynchrony task; $F(1,27)=28.32, M S_{\mathrm{e}}=161.95, p<.001$, for the intonation task]. In addition, the effect of target type was significant for the asynchrony task $\left[F(1,27)=8.37, M S_{\mathrm{e}}=\right.$ $71.17, p<.01]$, and the effect of harmonic context was significant for the intonation task $[F(1,27)=15.61$, $\left.M S_{\mathrm{e}}=63.57, p<.01\right]$. In both tasks, an interaction be- 
Asynchrony Task
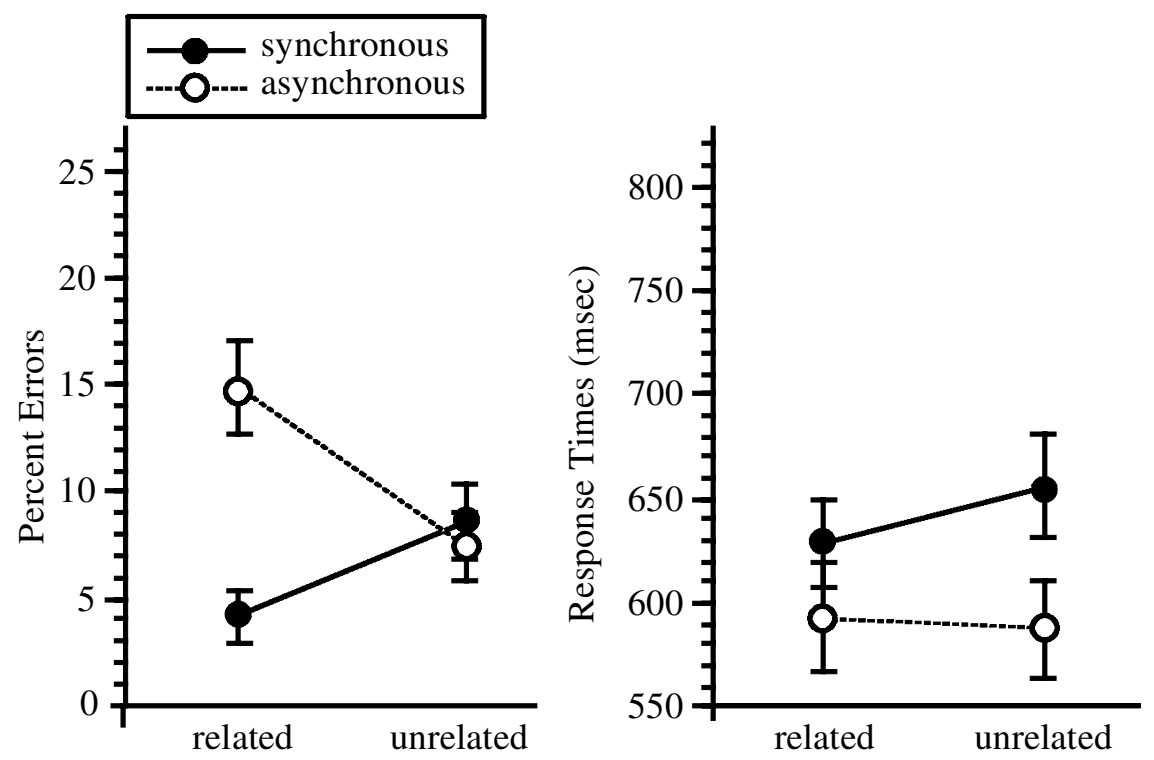

Intonation Task
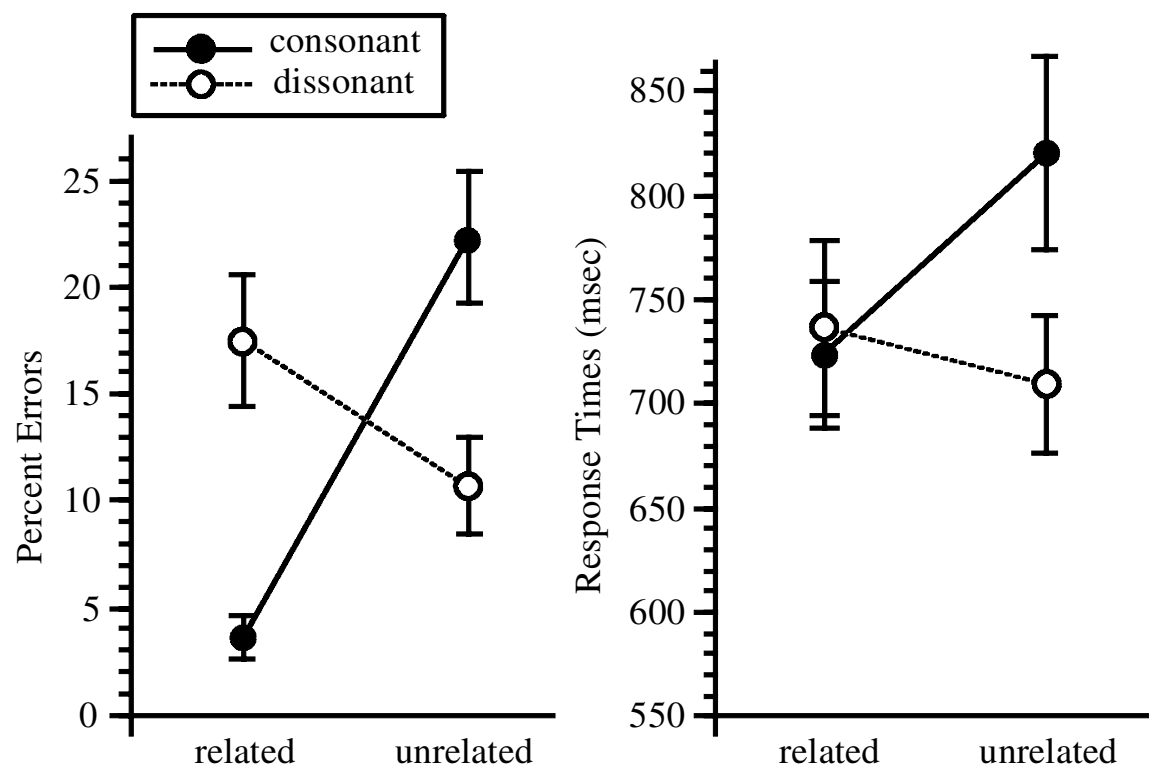

Figure 2. Percentages of errors (left panels) and correct response times (right panels) averaged across all chord pairs as a function of harmonic context (related/un related) and target type for the asynchrony task (top) and the intonation task (bottom) in Experiment 2. Error bars indicate between-subjects standard errors.

tween harmonic context and target type was observed. However, the error rate difference between the related and the unrelated contexts was more pronounced for consonant targets than for synchronous targets. In a $2 \times$ $2 \times 2$ ANOVA with task (asynchrony/intonation) as the third within-subjects factor, this difference in interaction found expression in a three-way interaction between task, harmonic context, and target type $[F(1,27)=6.67$, $\left.M S_{\mathrm{e}}=94.10, p<.05\right]$.
Response times. For each task (asynchrony/intonation), correct response times (Figure 2, right panels) were analyzed by a $2 \times 2$ ANOVA with harmonic context and target type as within-subjects factors.

For the asynchrony task, the influence of harmonic context was observed primarily for synchronous targets: Response times were shorter in the related context than in the unrelated context $\left[F(1,27)=5.07, M S_{\mathrm{e}}=2,082.79\right.$, $p<.05]$. The main effect of harmonic context and its 
interaction with target type failed to reach significance $(p<.09$ and $p=.08$, respectively). As was observed in Experiment 1, the main effect of target type was significant $\left[F(1,27)=19.53, M S_{\mathrm{e}}=3,895.8, p<.001\right]$ : Reaction times were shorter for asynchronous than for synchronous targets.

For the intonation task, the effects of harmonic context $\left[F(1,27)=10.01, M S_{\mathrm{e}}=3,328.5, p<.01\right]$ and of target type $\left[F(1,27)=6.18, M S_{\mathrm{e}}=10,797.2, p<.05\right]$ and their interaction $\left[F(1,27)=13.25, M S_{\mathrm{e}}=7,953.9, p<.01\right]$ were significant. For consonant targets, response times were shorter in the related than in the unrelated context. For dissonant targets, the reverse tendency was observed, with longer response times in the related context.

In both tasks, the size of the priming effect for synchronous and consonant targets (i.e., the difference in response times between related and unrelated contexts) was not correlated with musical training $[r(26)=-.01$ for the asynchrony task and $r(26)=.16$ for the intonation task].

Sensitivity and response criterion. Signal detection parameters $d^{\prime}$ and $c$ (Figure 3 ) were analyzed with two $2 \times 2$ ANOVAs, with task (asynchrony/intonation) and harmonic context (related/unrelated) as within-subjects factors. For sensitivity $\left(d^{\prime}\right.$; Figure 4, left), the interaction between task and harmonic context was significant $\left[F(1,27)=11.88, M S_{\mathrm{e}}=0.28, p<.001\right]$. This interaction confirmed the hypothesis, based on previous results, that the influence of context differs between the two tasks. As for long harmonic contexts (see Tillmann et al., 1998), $d^{\prime}$ for the intonation task was higher for related than for unrelated chords $[F(1,27)=23.6, p<.001]$. In contrast, as in Experiment 1, $d^{\prime}$ for the asynchrony task was higher for unrelated than for related chords [although here, this difference was not significant in a separate comparison;
$F(1,27)=1.11, p=.30]$. In addition, the main effects of task and of harmonic context were significant $[F(1,27)=$ $6.18, M S_{\mathrm{e}}=0.17, p<.05$, and $F(1,27)=7.1, M S_{\mathrm{e}}=0.55$, $p<.05$, respectively].

For the response criterion ( $c$; Figure 3, right panel), the effect of harmonic context and its interaction with task were significant $\left[F(1,27)=39.85, M S_{\mathrm{e}}=0.10, p<.001\right.$, and $F(1,27)=7.99, M S_{\mathrm{e}}=0.05, p<.01$, respectively]. There was a bias to answer one note lateldissonant for unrelated chords and a bias to answer normal/consonant for related chords. Both tasks showed a similar shift in response criterion, although it was stronger in the intonation task than in the asynchrony task. The analogous criterion shift in the two tasks suggests that the previously observed bias in the intonation task was not due only to a potential conflict between the types of dissonance (contextual dissonance and target dissonance), although it might reinforce the bias, but seems to reflect a more general phenomenon linked to contextual harmonic relatedness.

\section{Discussion}

In Experiment 2, harmonic priming was observed with both temporal asynchrony and intonation judgments. The processing of a (synchronous, consonant) target chord was facilitated when it was preceded by a related prime chord, in comparison with an unrelated prime chord. The fact that priming was observed for both tasks eliminates the possibility that priming effects observed with intonation judgments are due solely to a confound between dissonance types - the contextual dissonance created by harmonic unrelatedness and the dissonance of the target itself. Converging evidence has been reported recently with a phoneme-monitoring task for long context priming (Bigand, Tillmann, Poulin, \& D'Adamo, 2001). Lis-
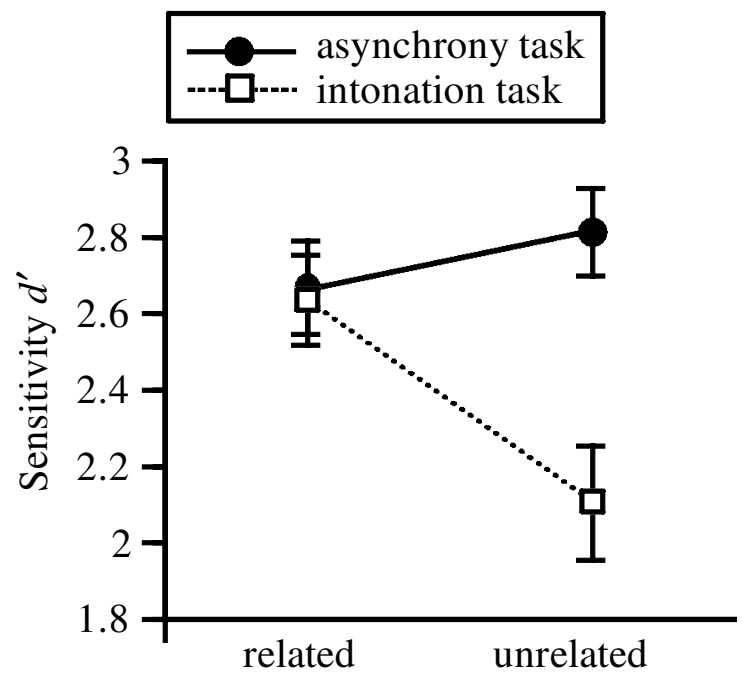

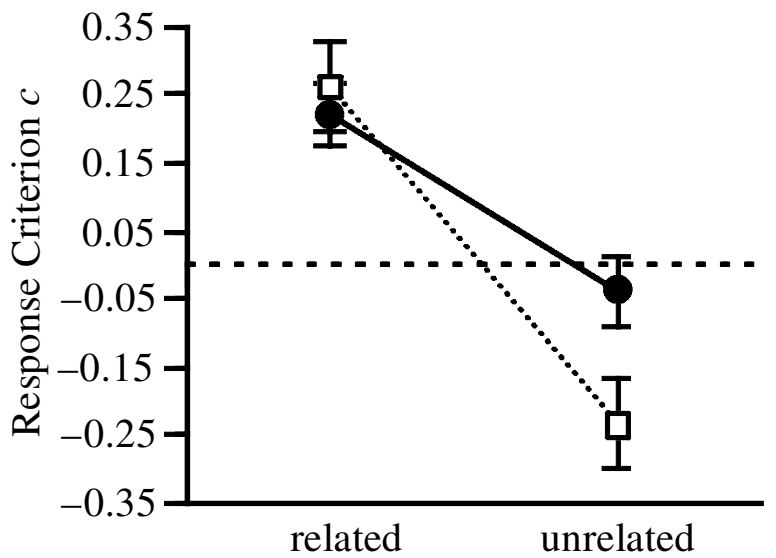

Figure 3. Left panel: sensitivity $\left(d^{\prime}\right)$ as a function of harmonic context (related/unrelated) and task (asynchrony/intonation). Right panel: response criterion (c) as a function of harmonic context (related/unrelated) and task (asynchrony/intonation). Positive values stand for a tendency to respond normal/consonant; negative values stand for a tendency to respond one note late/dissonant. Error bars indicate between-subjects standard errors. 


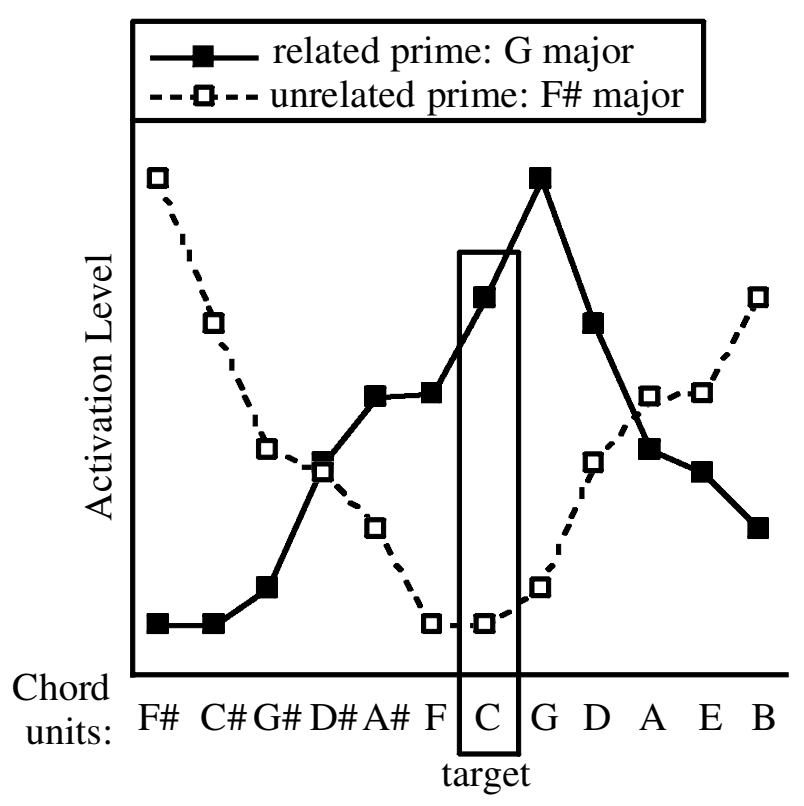

Figure 4. Relative activation of major chord units in the network at equilibrium after the presentation of a G-major and an $F \#$-major chord. The activation of the chord unit C-major is stronger after the harmonically related prime chord $G$ than after the unrelated chord $\mathbf{F}$.

tening to chord sequences created with synthetic phonemes, participants were required to decide quickly whether the target (the last chord of the sequence) was sung on the phoneme /i/ or /u/. Results showed that harmonic relatedness influences the processing of the target chord: Phoneme monitoring was faster for phonemes sung on a strongly related target (a referential tonic chord) than for those sung on a less related, structurally less important target (a subdominant chord). The priming results obtained with three different tasks thus point to the robustness of the processing advantages conferred by expectations arising from a previous context. This previous context can be as short as one single chord and influences the processing of further events. The outcome of the temporal asynchrony task showed that expectations based on harmonic structures of the context also have an impact on the perception of temporal features of an event.

\section{GENERAL DISCUSSION}

Our study provides evidence that a harmonic context influences the processing of a target chord: Temporal synchrony judgments and consonant judgments of target chords are facilitated after related prime chords, as compared with unrelated prime chords. In previous studies (Bharucha \& Stoeckig, 1986, 1987; Bigand et al., 1999; Bigand \& Pineau, 1997; Bigand, Poulin, et al., 2001; Bigand, Tillmann, et al., 2001; Tillmann \& Bigand, 2001;
Tillmann et al., 1998) and the present one, musical expertise only weakly influences priming. The fact that the harmonic context effect is observed consistently for musicians and for participants without musical training or formal knowledge in tonal music suggests that harmonic priming is based on cognitive processes that do not require explicit knowledge of musical structure. Listeners to Western music acquire, through mere exposure, implicit knowledge of the tonal system and its underlying regularities. The acquisition of implicit tonal knowledge and the activation of this knowledge in a context can be simulated by a connectionist model (Bharucha, 1987; Tillmann et al., 2000). The model of tonal knowledge activation simulates previously observed harmonic priming effects (Bharucha \& Stoeckig, 1987; Bigand et al., 1999; Tekman \& Bharucha, 1998; Tillmann \& Bigand, 2001; Tillmann et al., 1998). For our data, it provides a framework to explain the short context priming and the sensitivity differences between the two tasks, depending on the context.

In the model, tonal knowledge is conceived as a network of interconnected units. Once learning has occurred, the units are organized in three layers corresponding to tones, chords, and keys. Each tone unit is connected to the chords of which that tone is a component. Analogously, each chord unit is connected to the keys of which it is a member. Harmonic relations emerge from the activation that reverberates via connected links between tone, chord, and key units. When a chord is played, the units representing the sounded component tones are activated, and phasic activation (i.e., the change of activation) is sent via connected links from tones to chords and from chords to keys (bottom-up activation). Phasic activation reverberates from keys to chords and from chords to tones (top-down activation) until an equilibrium is reached (see Bharucha, 1987, and Tillmann et al., 2000, for more details). In early activation cycles, activated chord units contain at least one of the component tones of the stimulus chord. At equilibrium, the activation pattern of chord units and of tone units reflects the Western tonal hierarchy of the key context and takes into account the key membership of chords and tones. A given chord activates the units of harmonically related chords more strongly than it does the units of unrelated chords. After two harmonically distant prime chords (e.g., G and F\#-major in Figure 4), the activation patterns differ strongly, with each one reflecting its harmonic relations with the other major chords. A target chord unit (e.g., C-major) is more strongly activated after the presentation of a harmonically related chord that shares a parent key (G-major) than after an unrelated chord with no common parent key (F\#-major). Activation levels are interpreted as levels of expectation for subsequent events (Bharucha \& Stoeckig, 1987; Bigand et al., 1999; Tekman \& Bharucha, 1998; Tillmann et al., 1998) and predict harmonic priming, with facilitated processing after a related prime. In our study, activation levels of 
chord units thus simulate the observed harmonic context effects on correct response times and error rates for synchronous and consonant targets.

However, for the asynchronous and dissonant targets, the pattern of results (primarily of the accuracy data) differed between the two tasks. Signal detection analysis revealed that this difference was not due to the response criterion, but rather to sensitivity: $d^{\prime}$ was higher for the unrelated context than for the related context in the temporal asynchrony task (Experiments 1 and 2), and the reverse was observed in the intonation task (Experiment 2). The question arises as to how the neural net model of tonal knowledge activation can account for these observed context differences in $d^{\prime}$.

The asynchrony and intonation tasks involve the processing of a particular tone in the target chord: the delayed tone and the tone creating the dissonance. When a chord is presented to the model, activation reverberates to chord and key layers and back to the tone layer, where the received phasic activation reflects the importance of the tones in the actual key context (i.e., the tonal hierarchy). After the presentation of a G-major chord (i.e., one consisting of the three tones $\mathrm{G}-\mathrm{B}-\mathrm{D})$, the activation of the tone layer reflects the relationship among tones in the key of G-major-even if not all tones have been presented to the model (cf. profile of the related prime Gmajor in Figure 5). As a consequence of top-down influences in reverberation, the tone units belonging to the key of G-major (A, B, C, D, E, F\#, G) receive more activation than do tone units outside the key $(A \#, C \#, D \#, F$, $G \#)$, with a reduced activation for the tone $F \#$.

In response to different prime chords (i.e., $\mathrm{G}$ and $\mathrm{F \# -}$ major), the activation patterns of the tone units reflect the tonal relations among the 12 tones in the corresponding

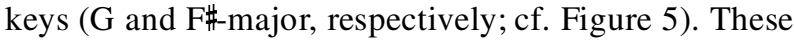
activation levels determine how strong the change of activation in a tone unit will be when a particular tone occurs in the following chord. If a tone unit is strongly activated by the previous context, the change of activation in this tone unit will be less than if it was weakly activated previously. When the listeners' task involves a detection of this tone, we hypothesize that the strength of activation change influences the ease of tone detection: A small activation change might result in a more difficult task and less precise processing than does a large activation change. In addition, a strong activation level of a tone unit before the tone sounds (or even if it does not occur) might render the rejection of its presence in the following chord more difficult than does a weak activation level.

Because the sensitivity data differ as a function of harmonic context and task, we would expect different activation levels of these two tones as a function of the prime context, which in turn lead to different activation changes when the target occurs. For the example in Figure 5, the target that follows the related (G-major) and the unrelated (F\#-major) primes is a $\mathrm{C}$-major chord. In the asynchrony task, the to-be-detected tone in the target is the tone $\mathrm{C}$. Because of reverberation back to the tone layer, the activation level of the $\mathrm{C}$ unit is stronger after the related prime than after the unrelated prime. After the related prime, the delayed tone is already strongly activated by top-down reverberation before actually sounding. This strong activation and the resulting small activation change when the tone is then played in the target might render the detection of the tone (i.e., its moment of occurrence) more difficult than in the unrelated context, in which a low activation level is followed by a large activation change. In the intonation task, the distinctive tone

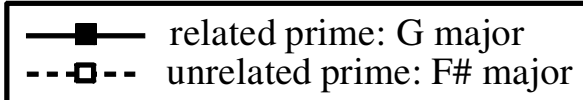

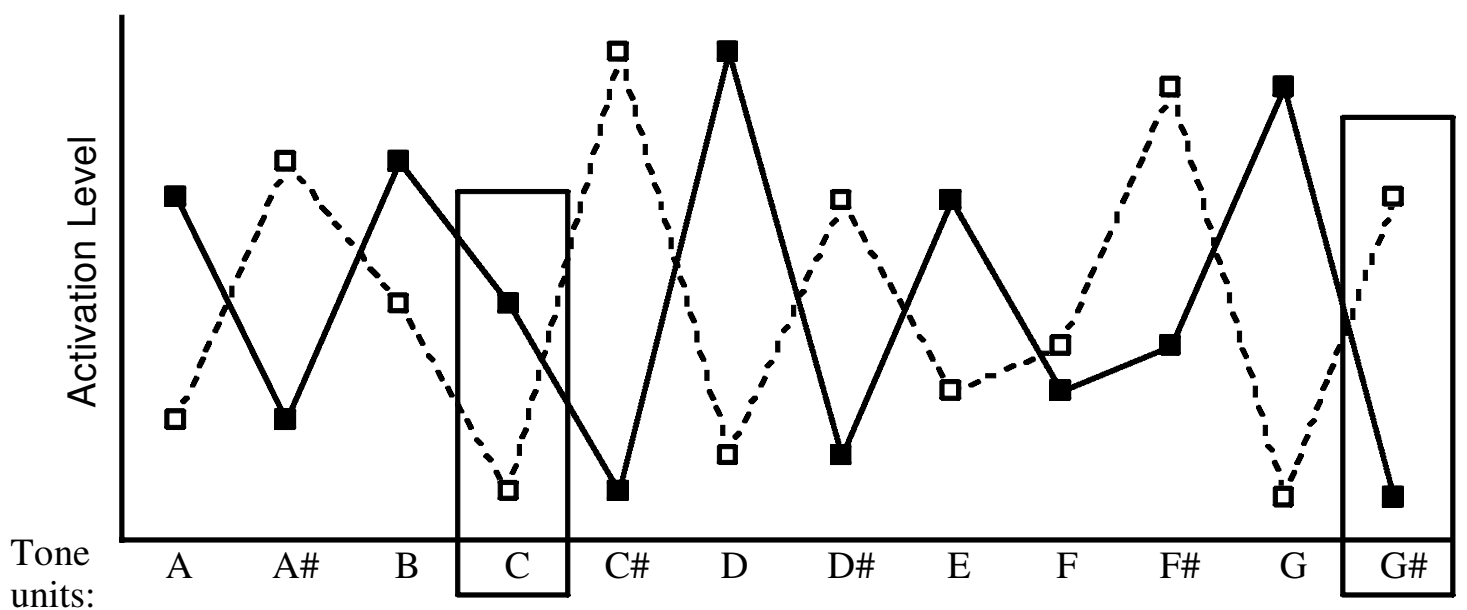

Figure 5. Activations received by the 12 units of the tone layer during reverberation after the presentation of a $\mathrm{G}$ major and an $\mathbf{F} \#$-major chord. 


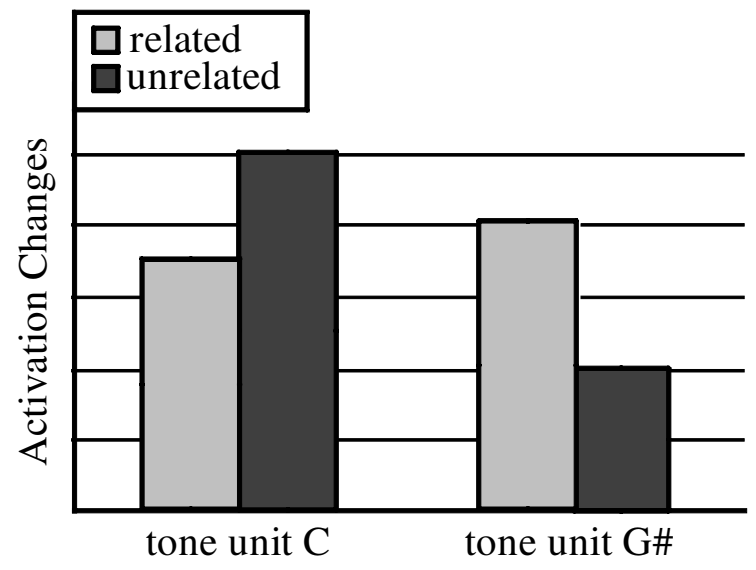

Figure 6. Difference of activation for the tone units $\mathbf{C}$ and $\mathbf{G} \#$ before and after the presentation of the chord $\mathrm{C}$-major that followed the prime chord (i.e., the related G-major or the unrelated $F \#$ major chord). The tone $C$ is the crucial feature for the asynchrony task, and the tone $G \sharp$ for the intonation task.

creating the dissonance is $\mathrm{G} \#$. It is more strongly activated after an unrelated prime than after a related prime. The activation levels are thus reversed in comparison with the tone $\mathrm{C}$ and would result in the opposite activation change when the target is played. These activation patterns predict a reversed detection facilitation as a function of harmonic context than in the asynchrony task. The prediction for activation changes in tone units was strengthened by further simulations that were performed with the related and unrelated primes followed by either a C-major chord (consisting of the tones $\mathrm{C}-$ E-G) or a C-major chord with an additional G\# tone. ${ }^{2}$ Figure 6 displays the difference of activation before and after the presentation of the target for the tone units $\mathrm{C}$ and $\mathrm{G \#}$. For the tone $\mathrm{C}$, the activation change is stronger after the unrelated prime than after the related prime. For the tone $\mathrm{G} \#$, this pattern is reversed. The strength of activation change thus depends on the harmonic context and the considered tone, and it reflects the pattern of the $d^{\prime}$ data observed for the asynchrony and intonation tasks.

The simulations suggest that the neural net model of tonal knowledge activation provides a framework for integrating the observed priming results of the two tasks. The facilitated processing of normative (synchronous, consonant) targets (with higher accuracy and faster response times) is simulated at a global level of processing by the activation of the target chord unit. The differences in $d^{\prime}$ between the two tasks depending on the harmonic context are simulated with differences in activation levels and activation changes of the tone units that represent the critical features. The model explains the influence of harmonic context on the detection of temporal onset asynchrony in terms of activation of listeners' implicit knowledge of harmonic relations. The model also offers a framework for generating new, testable predic- tions that relate to the influence of harmonic relations on the processing of temporal features. A specific prediction derives directly from activation levels in tone units (Figure 5): A similar outcome should occur when the delayed tone in the target is not $\mathrm{C}$, but $\mathrm{G}$ or $\mathrm{E}$. Further predictions are linked to the temporal decay of activation, which simulates the dynamic aspects of harmonic processing. Systematic modifications of the interstimulus interval should allow us to further investigate the time course of knowledge activation and its influence on temporal processing.

The present study suggests that structures based on the pitch dimension influence the processing of temporal information. The harmonic relatedness of the context chord influences the detection of a temporal onset asynchrony in simultaneously occurring tones. A further concern is to investigate whether these results generalize to temporal processing in longer contexts. It already has been shown that the temporal perception of sequentially occurring events in musical sequences is influenced by the overall phrase structure of the piece (Repp, 1992, 1998, 1999). Future research must investigate whether the detection of temporal deviations between sequential musical events is influenced specifically by the harmonic relatedness of the deviant event to its surrounding context. For example, the question arises whether listeners' sensitivity to accurate timing is smaller for strongly related events than for unrelated ones, or whether a deviant tone would be more easily detected when it is a nondiatonic event —or even a less related diatonic eventin the actual key context.

\section{REFERENCES}

BharuCha, J. J. (1987). Music cognition and perceptual facilitation: A connectionist framework. Music Perception, 5, 1-30.

Bharucha, J. J., \& Stoeckig, K. (1986). Reaction time and musical expectancy: Priming of chords. Journal of Experimental Psychology: Human Perception \& Performance, 12, 403-410.

Bharucha, J. J., \& StoecKig, K. (1987). Priming of chords: Spreading activation or overlapping frequency spectra? Perception \& Psychophysics, 41, 519-524.

Bigand, E., Madurell, F., Tillmann, B., \& Pineau, M. (1999). Effect of global structure and temporal organization on chord processing. Journal of Experimental Psychology: Human Perception \& Performance, 25, 184-197.

Bigand, E., \& Pineau, M. (1997). Global context effects on musical expectancy. Perception \& Psychophysics, 59, 1098-1107.

Bigand, E., Poulin, B, Tillmann, B., D’ Adamo, D., \& Madurell, F. (2001). Cognitive versus sensory components in harmonic priming. Manuscript submitted for publication.

Bigand, E., Tillmann, B., Poulin, B., \& D'Adamo, D. (2001). The effect of harmonic context on phoneme monitoring in vocal music. Cognition, 81, B11-B20.

Bregman, A. S. (1990). Auditory scene analysis: The perceptual organization of sound. Cambridge, MA: MIT Press.

Cohen, J., MacWhinney, B., Flatt, M., \& Provost, J. (1993). PsyScope: An interactive graphic system for designing and controlling experiments in the psychology laboratory using Macintosh computers. Behavior Research Methods, Instruments, \& Computers, 25, 257-271.

Drake, C. (1993). Perceptual and performed accents in musical sequences. Bulletin of the Psychonomic Society, 31, 107-110. 
Drake, C., \& Botte, M.-C. (1993). Tempo sensitivity in auditory sequences: Evidence for a multiple-look model. Perception \& Psychophysics, 54, 277-286.

FrAnCÈs, R. (1958). La perception de la musique. Paris: Vrin. [The perception of music (W. J. Dowling, Trans.) (1988). Hillsdale, NJ: Erlbaum]

Halpern, A. R. \& Darwin, C. J. (1982). Duration discrimination in a series of rhythmic events. Perception \& Psychophysics, 31, 86-89.

Hirsh, I. J., Monahan, C. B., Grant, K. W., \& Singh, P. G. (1990). Studies in auditory timing: I. Simple patterns. Perception \& Psychophysics, 47, 215-226.

KADLEC, H. (1999). Statistical properties of $d^{\prime}$ and $\beta$ estimates of signal detection theory. Psychological Methods, 4, 2-43.

Krumhansl, C. L. (1990). Cognitive foundations of musical pitch. Oxford: Oxford University Press.

Macmillan, N. A., \& Creelman, C. D. (1991). Detection theory: A user's guide. Cambridge: Cambridge University Press.

Meyer, D. E., \& Schvaneveldt, R. W. (1971). Facilitation in recognizing pairs of words: Evidence of a dependence between retrieval operations. Journal of Experimental Psychology, 90, 227-234.

PALmer, C. (1996). On the assignment of structure in music performance. Music Perception, 14, 23-56.

REPP, B. H. (1992). Probing the cognitive representation of musical time: Structural constraints on the perception of timing. Cognition, 44, 241-281.

REPP, B. H. (1998). Variations on a theme by Chopin: Relations between perception and production of timing in music. Journal of Experimental Psychology: Human Perception \& Performance, 24, 791-811.

REPP, B. H. (1999). Detecting deviations from metronomic timing in music: Effects of perceptual structure on the mental timekeeper. Perception \& Psychophysics, 61, 529-548.

Stanovich, K. E., \& WeSt, R. F. (1979). Mechanisms of sentence context effects in reading: Automatic activation and conscious attention. Memory \& Cognition, 7, 77-85.

Tekman, H. G., \& Bharucha, J. J. (1992). Time course of chord priming. Perception \& Psychophysics, 51, 33-39.

Tekman, H. G., \& Bharucha, J. J. (1998). Implicit knowledge versus psychoacoustic similarity in priming of chords. Journal of Experimental Psychology: Human Perception \& Performance, 24, 252-260.
Terhardt, E. (1984). The concept of musical consonance: A link between music and psychoacoustics. Music Perception, 1, 276-295.

Tillmann, B., Bharucha, J. J., \& Bigand, E. (2000). Implicit learning of music: A self-organizing approach. Psychological Review, 107, 885-913.

Tillmann, B., \& Bigand, E. (2001). Global context effect in normal and scrambled musical sequences. Journal of Experimental Psychology: Human Perception \& Performance, 27, 1185-1196.

Tillmann, B., Bigand, E., \& Pineau, M. (1998). Effects of global and local contexts on harmonic expectancy. Music Perception, 16, 99118.

Zera, J., \& Green, D. M. (1993). Detecting temporal onset and offset asynchrony in multicomponent complexes. Journal of the Acoustical Society of America, 93, 1038-1052.

ZERA, J., \& GREen, D. M. (1995). Effect of signal component phase on asynchrony discrimination. Journal of the Acoustical Society of America, 98, 817-827.

\section{NOTES}

1. Error rates and response times were reported side by side to show facilitation (fewer errors and shorter reaction times) and to show that the observed context effect was not due solely to a speed/accuracy tradeoff.

2. The model in its present form does not include pitch height. The 12 chromatic tone units represent absolute pitch classes only (i.e., tones generalized across octaves). Because of this representation, the C-major chord was coded with three tones (each tone was coded in the input layer with an activation value of 1 ). The additional tone $\mathrm{G} \#$ was coded with a value of .25 , in order to have a comparable energy input for the two presented chords (i.e., $\mathrm{C}-\mathrm{E}-\mathrm{G}$ and $\mathrm{C}-\mathrm{E}-\mathrm{G}-\mathrm{G} \#$ ). It should be noted that the absolute amount of change (Figure 6) cannot be compared across units, because this relation depends on the coded input value for

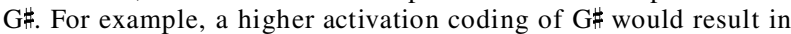
stronger activation changes for $\mathrm{G} \#$ than occur with the present coding and that would be stronger than those for the tone $\mathrm{C}$.

(Manuscript received October 23, 2000; revision accepted for publication August 1, 2001.) 